\title{
Fertilidad y fallas reproductivas en un rebaño de cabras criollas en el trópico subhúmedo, sincronizadas con esponjas vaginales.
}

\section{Artículo Original}

Antonio Ortega-Pacheco ${ }^{1}$, Juan F. de J. Torres-Acosta ${ }^{1}$, Armando J. Aguilar-Caballero' ${ }^{1}$, Julio P. RamónUgalde $^{2}$.

${ }^{1}$ Universidad Autónoma de Yucatán, Facultad de Medicina Veterinaria y Zootecnia, Mérida, Yucatán, ${ }^{2}$ Instituto Técnico Agropecuario No. 2, Conkal, Yucatán, México.

\section{RESUMEN.}

Introducción. El comportamiento reproductivo de las cabras en el trópico presenta altos índices de fertilidad. La alta temperatura ambiental y disponibilidad de forrajes pueden afectar este comportamiento. La sincronización de estros mediante progestágenos se emplea para manipular la actividad sexual pero puede afectar la eficiencia reproductiva. El objetivo de este trabajo fue determinar la fertilidad y tipo de falla reproductiva en un rebaño de cabras criollas después de sincronizar sus celos empleando progestágenos por vía vaginal, gonadotrofina coriónica equina (eCG) y monta natural.

Material y métodos. Se sincronizó el estro de 33 cabras criollas empleando esponjas vaginales con $40 \mathrm{mg}$ de acetato de flurogestona más eCG y se permitió la monta. Se determinó el número de ovulaciones contando mediante laparoscopia el número de cuerpos luteos. Se determinó la gestación mediante ultrasonografía 45 días postservicio (PS). La eficiencia y porcentaje de fallas reproductivas fueron medidas después de los partos.

Resultados. El 94\% de las cabras mostró estro; $43.20 \%$ se encontraron gestantes a 45 días PS; $33.335 \%$ parieron con una prolificidad de 2.4 crías. La tasa ovulatoria del total de las cabras fue de 2.97; las hembras paridas y no paridas tuvieron tasas ovulatorias de 3.62 y 2.50 respectivamente. Una hembra presentó anestro (2.94\%) y anovulación (2.94\%). Las fallas de fertilización y/ o muerte embrionaria fueron de $52.94 \%$ en hembras paridas y $76.24 \%$ incluyendo las ovulaciones de hembras no paridas. Tres abortos se detectaron $(9.1 \%)$.

Solicitud de sobretiros: Antonio Ortega-Pacheco. Facultad de Medicina Veterinaria y Zootecnia, Universidad Autónoma de Yucatán. Km 15.5 carretera Mérida-Xmatkuil, A.P. 4-116, Itzimná, Mérida, Yucatán, México. 
A Ortega-Pacheco, JF de JTorres-Acosta, AJ Aguilar-Caballero, JP Ramón-Ugalde.

Discusión. La terapia empleada fue eficiente para la inducción y sincronización de estros. La tasa de gestación y fertilidad fue muy baja probablemente por la alta incidencia de muerte embrionaria y abortos ocurridos. La prolificidad fue consecuencia de la alta tasa ovulatoria. La temperatura ambiental y competencia embrionaria probablemente causaron la muerte embrionaria.

(Rev Biomed 2002; 13:179-184)

Palabras clave: Cabras, temperatura, fertilidad, muerte embrionaria.

\section{SUMMARY.}

Fertility and reproductive failure of criollo goats synchronized with vaginal sponges in the subhumid tropics.

Introduction. Reproductive performance of goats in the tropics shows high fertility rates. High temperature and forage availability can affect this behavior. Estrus synchronization by progestagens is used to control sexual activity but reproductive efficiency can be negatively affected.

The objective of this study was to determine fertility and reproductive failures in a flock of criollo goats after estrus synchronization using intravaginal progestagens, equine chorionic gonadotrophin (eCG) and natural mating.

Material and methods. Estrus of 33 criollo goats was synchronized using 40-mg flurogestone acetate vaginal sponges and eCG; natural service was allowed. Ovulation rate was determined by counting through laparoscopy, the number of corpus luteum. Pregnancy rate was determined by ultrasonography at 45 days postservice (PS). Reproductive efficiency and percentage of reproductive failure was determined after kidding occurred.

Results. From the goats, $94.11 \%$ showed estrus; $43.20 \%$ were pregnant at 45 days PS; $33.335 \%$ kidded and had a prolificy of 2.4 kids. Ovulation rate was 2.97; the ovulation rate for kidded and non kidded does was 3.62 and 2.50 respectively.
One doe did not showed estrus (2.94\%) and ovulation $(2.94 \%)$. Fertilization failure and embryonic death from kidded does was $52.94 \%$ and $76.24 \%$ including ovulations from non kidded does. Three abortions were detected $(9.1 \%)$.

Discussion. The therapy used was effective to induce and synchronized estrus. Pregnancy rate and fertility was very low probably because of the high incidence of embryonic death and abortions occurred. Prolificy was a consequence of high ovulation rate. Ambiental temperature and embryo competition were probably the cause of embryonic death. (Rev Biomed 2002; 13:179-184)

Key words: Goats, temperature, fertility, embryonic death.

\section{INTRODUCCIÓN.}

En condiciones tropicales, las cabras mantenidas bajo sistemas de empadre natural generalmente presentan altos índices de fertilidad y partos gemelares (1). Aunque el fenómeno de la estacionalidad reproductiva en los trópicos no esta bien definido, existe cierta tendencia de las cabras a presentar mayor actividad reproductiva en ciertos períodos del año, cuando las condiciones del medio ambiente son más favorables, para el mantenimiento de la gestación y cuidados de la cría. Se ha observado en borregas, que el estrés térmico y la poca disponibilidad y calidad de forrajes son factores que influyen grandemente en el desempeño reproductivo, de manera que, dentro de una época de empadre se presenta en mayor o menor frecuencia fallas reproductivas en un gran número de animales en los primeros servicios $(2,3)$.

La sincronización de celos empleando progestágenos es una práctica de uso común para el control reproductivo de los rebaños caprinos (4). Existe sin embargo evidencia de que la fertilidad subsiguiente sea reducida a pesar de la alta efectividad en la sincronización y aparición de celos $(4,5)$. No se sabe con exactitud cuáles sean las causas de estas fallas reproductivas, pero se ha

\section{Revista Biomédica}


Fertilidad y fallas reproductivas en cabras criollas sincronizadas con esponjas vaginales.

sugerido, entre otros, que la asincronía entre las relaciones embrión-útero, defectos genéticos (6), cuerpos lúteos poco viables y fallas en la fertilización $(4,6)$ pueden tener un papel importante en este problema.

El objetivo de este trabajo fue determinar la fertilidad, caracterizar y estimar el tipo y frecuencia de fallas reproductivas en un rebaño de cabras criollas mantenidas en condiciones del trópico subhúmedo, después de un programa de sincronización de celos empleando progestágenos por vía vaginal y monta controlada.

\section{MATERIALES Y MÉTODOS.}

Se utilizaron 33 cabras criollas adultas pertenecientes al área de Producción de Pequeños Rumiantes de la FMVZ-UADY, ubicado en la zona centro del estado de Yucatán, México. El tipo de clima es cálido subhúmedo con lluvias en verano. El trabajo se desarrolló durante los meses de abril a septiembre de 1998.

Los celos fueron sincronizados en el mes de abril empleando esponjas vaginales impregnadas con $40 \mathrm{mg}$ de acetato de fluorogestona (FGA) (Chronogest, Intervet Holland) por 14 días, seguido de 400 UI vía I.M. profunda de gonadotropina coriónica equina (eCG) (Folligon, Intervet Holland) al momento de retiro de las esponjas. Las esponjas fueron colocadas utilizando un espéculo vaginal previamente desinfectado y lubricado el cual se insertó en la vagina y se depositó la esponja en el tiempo de un minuto por cada animal. Al momento de aplicación de las esponjas los animales se encontraban, de acuerdo a sus registros, en anestro. Los celos fueron detectados por simple observación con ayuda de un macho marcador y se permitió la monta controlada al momento de la aceptación y 12 horas después, empleando un macho de fertilidad probada.

Se realizó a cada animal un examen laparoscópico a los 8 días postservicio (PS) con la finalidad de contar el número de cuerpos lúteos (CL) presentes en cada ovario. Para esto se colocó al paciente en posición decúbito dorsal. Previa asepsia de la piel, se introdujo en cavidad abdominal un laparoscopio por medio de un trocar colocado a través de una incisión a lo largo de la línea media. Se hizo una infusión por la cánula de $\mathrm{CO} 2$ al 5\% en aire dentro de cavidad abdominal y se procedió a la observación.

El diagnóstico de gestación se realizó a los 45 días PS mediante ultrasonografía de tiempo real. Se empleó la técnica transrectal con el animal en pie empleando un equipo modelo Vet 200 Pie Medical equipado con un transductor sectorial de $7.5 \mathrm{MHz}$.

Se utilizó estadística descriptiva para reportar el porcentaje de cabras que manifestaron celo, la tasa ovulatoria, tasa de gestación a los 45 días PS, la fertilidad (No. hembras tratadas/No. hembras paridas), la prolificidad (No. crías nacidas/No. hembras paridas) y frecuencia de fallas reproductivas (anestro, anovulación, fallas en fertilización y/o muerte embrionaria y tasa de abortos). Las fallas en la fertilización y/o muerte embrionaria se determinaron comparando para cada cabra parida, el número de cabritos nacidos con el número de cuerpos lúteos observados en ambos ovarios 8 días PS. Las fallas de fertilización y/o muerte embrionaria totales se calcularon considerando el mismo criterio señalado pero incluyendo también los CL de las cabras no paridas.

\section{RESULTADOS.}

Los resultados de fertilidad y demás eventos reproductivos del rebaño después de la terapia con FGA y eCG se observan en el cuadro 1.

Se observa que de las 33 cabras tratadas, 32 de ellas manifestaron signos de celo, las cuales todas fueron montadas al menos en dos ocasiones durante el mismo celo. La tasa de gestación determinada por ultrasonografía a los 45 días PS fue del $43.30 \%$. Solamente 10 cabras parieron un total de 24 crías viables, lo que representó el $33.33 \%$ de fertilidad y 2.4 crías nacida por hembra de prolificidad.

La tasa ovulatoria medida por el número de 
A Ortega-Pacheco, JF de J Torres-Acosta, AJ Aguilar-Caballero, JP Ramón-Ugalde.

Cuadro 1

Porcentaje de celos, montas, tasa de gestación a 45 días, fertilidad y prolificidad en un rebaño de cabras criollas sincronizadas con FGA* y eCG $\dagger$.

\begin{tabular}{lcr}
\hline \multicolumn{1}{c}{ Evento } & No. de observaciones & $\%$ \\
\hline aparición de celos & 32 hembras & 94.11 \\
montas hembra/celo & 32 hembras & 100.00 \\
Tasa de gestación a & 13 hembras gestantes & 43.30 \\
45 días & & \\
fertilidad & 10 hembras paridas & 33.33 \\
prolificidad & 24 crías nacidas & 2.4 crías \\
& & nacidas/hembra \\
& &
\end{tabular}

* FGA = Acetato de fluorogestona

$\dagger$ eCG = Gonadotropina coriónica equina determinó la causa exacta de los abortos pero al parecer se debieron a traumatismos.

\section{DISCUSIÓN.}

El porcentaje de aparición y de sincronización de celos encontrado en este trabajo fue alto (94.11\%) de igual manera como se ha reportado en otros trabajos $(7,8,9)$. Esto indica que la terapia empleada con FGA y eCG fue efectiva, considerando también que el $100 \%$ de las hembras en celo fueron servidas.

La tasa de gestación de 45 días PS (43.30\%) fue similar a lo señalado en algunos reportes (10),

Cuadro 2

Tasa ovulatoria en hembras paridas y no paridas en un rebaño de cabras criollas sincronizadas con FGA* y eCG $\dagger$.

\begin{tabular}{lllll}
\hline & $\mathrm{n}$ & Ovario Der. & Ovario Izq. & Total \\
\hline Hembras paridas & 10 & $2.14(\mathrm{n}=30)$ & $1.50(\mathrm{n}=21)$ & $3.64(\mathrm{n}=51)$ \\
Hembras no paridas & 23 & $1.25(\mathrm{n}=25)$ & $1.25(\mathrm{n}=25)$ & $2.50(\mathrm{n}=50)$ \\
Total & 33 & $1.62(\mathrm{n}=55)$ & $1.35(\mathrm{n}=46)$ & $2.97(\mathrm{n}=101)$ \\
\hline
\end{tabular}

* FGA = Acetato de fluorogestona

$\dagger$ eCG = Gonadotropina coriónica equina

CL presentes en los ovarios de las cabras sincronizadas se observa en el cuadro 2.

Las hembras paridas tuvieron un total de 51 cuerpos lúteos lo que representó una tasa ovulatoria de 3.64 vs. 2.50 encontrado en las hembras no paridas. El ovario derecho en ambos grupos de hembras fue más activo que el izquierdo, observándose un total de 55 ovulaciones lo que representa una tasa ovulatoria de 1.62.

La frecuencia encontrada y tipo de fallas reproductivas se observan en el cuadro 3 .

Una cabra presentó anestro y no ovuló (2.94\%). Las fallas en la fertilización y/o muerte embrionaria en las cabras paridas fue de $27 \mathrm{CL}$ de 51 sin producir cría lo cual representó el 52.94\%. Por otro lado, considerando también las ovulaciones ocurridas en las cabras no paridas, este tipo de falla representó el 76.24\%. Se detectaron 3 abortos de gestaciones a término. No se

\section{Revista Biomédica}

pero por abajo al $93.75 \%$ señalado por otros (11) en cabras criollas con celos sincronizados

\section{Cuadro 3}

Frecuencia y tipo de falla reproductivas encontradas en un rebaño de cabras criollas sincronizadas con FGA* y eCG $\dagger$.

\begin{tabular}{lcc}
\hline Tipo de falla & n & $\%$ \\
\hline Anestro & 1 & 2.94 \\
Anovulación & 1 & 2.94 \\
Fallas en fertilización & $27(\mathrm{CL}) \$ 52.94$ \\
y/o muerte embrionaria & & \\
en hembras paridas & & \\
Fallas en fertilización & 77 (CL) 76.24 \\
y/o muerte embrionaria & & \\
total & & \\
Abortos & 3 & 9.1 \\
\hline
\end{tabular}

\footnotetext{
* FGA = Acetato de fluorogestona

$\dagger$ eCG = Gonadotropina coriónica equina

$\ddagger \mathrm{CL}=$ Cuerpo lúteo
} 


\section{Fertilidad y fallas reproductivas en cabras criollas sincronizadas con esponjas vaginales.}

empleando progestágenos. La fertilidad (33.33\%) consecuentemente estuvo también por debajo de lo esperado empleando FGA más eCG $(4,5,12)$. Por otro lado, la prolificidad (2.4 crías nacidas hembra/parto) fue superior a lo esperado en cabras criollas bajo programas de sincronización de celos (4) o bajo condiciones de empadre continuo (13).

El anestro y la anovulación que se presentó en una cabra fueron posiblemente consecuencia de un estado de desbalance hormonal o estados de tensión lo cual en ocasiones evitan una buena respuesta al tratamiento (14).

Las pérdidas de ovocitos considerando el número de CL observados y comparándolos con el número de crías nacidas, ha sido usado en otros estudios como indicador de que no ocurrió la concepción o de que se perdió el embrión tempranamente $(15,16)$. En este trabajo, en las hembra paridas, las fallas reproductivas en este sentido fueron muy altas $(52.94 \%)$. La tasa ovulatoria (3.64) y prolificidad (2.40) encontrada en este grupo señalan que la mayoría de los ovocitos liberados estuvieron en situación de fecundarse; de haberse dado esta situación, por alguna razón muchos de los nuevos productos formados no fueron viables. Ante esto se ha señalado que es en los períodos tempranos de gestación donde ocurre gran cantidad de pérdidas embrionarias (hasta un 10\%) por defectos genéticos de embrión ovino (6). Por otro lado, se ha reportado en borregas la existencia de una relación inversa entre la tasa ovulatoria y la proporción de embriones que sobreviven. Ocurre mayor muerte embrionaria en borregas con más ovulaciones por deficiencias en su desarrollo al haber un amplio intervalo entre ovulaciones o por defectos de migración uterina (17). Se ha observado que antes del día 20 de gestación puede ocurrir muerte embrionaria en 10-15\% de hembras con gestaciones simples y del 10-25\% en hembras con dos embriones (2). El estrés calórico incrementa también grandemente la mortalidad embrionaria sobre todo antes del día 20 de gestación, esto probablemente a la inducción de anomalías cromosómicas durante el reinicio de la meiosis (3). Las altas temperaturas registradas durante la primavera y verano en Yucatán, México, probablemente jueguen algún papel en la mortalidad ocurrida en este trabajo.

Por otro lado, se ha señalado que la exposición de los folículos dominantes a concentraciones variables de progesterona es importante en la fertilidad posterior a la ovulación, sobre todo en programas de sincronización de celos empleando progestágenos (18). Esto es quizá debido a un desarrollo anormal del oocito, de tal manera que su posible fertilización o subsiguiente desarrollo embrionario se vea comprometido o que se altere el medio ambiente del oviducto o uterino que evite el transporte espermático y/o subsiguiente desarrollo fetal (3).

La reducción del 10\% en la tasa de gestación entre el día 45 de gestación y el parto fue un evento que redujo grandemente la fertilidad en el programa de sincronización de celos. Hacia el día 45 de gestación, los fetos pueden morir por una gran variedad de condiciones; al suceder esto, los productos son reabsorbidos lentamente, son momificados o generalmente son abortados. En este trabajo solamente tres abortos fueron diagnosticados; sin embargo al salir los animales a ramonear en los agostaderos de alrededor, otros probables abortos no fueron vistos. En borregas, la proporción de productos perdidos después del día 30 de gestación puede variar de $2-9 \%$ en gestaciones simples, del $1-11 \%$ en gestaciones gemelares y del 5-13\% en gestaciones triples (2).

Las fallas en la fertilización y/o muerte embrionaria total (76.24\%), considerando a las hembras no paridas señala que este tipo de fallas reproductivas puede ser todavía mayor de lo que se ha reportado.

Se concluye que la sincronización de celos en cabras criollas mediante FGA y eCG bajo condiciones del trópico es efectiva, pero existe gran porcentaje de fallas reproductivas debido probablemente a fallas en la fertilización y/o muerte embrionaria. El posible papel de la temperatura ambiental se 


\section{A Ortega-Pacheco, JF de J Torres-Acosta, AJ Aguilar-Caballero, JP Ramón-Ugalde.}

considera como un factor importante en estas fallas.

\section{REFERENCIAS.}

1.- Jaramillo LE. Comportamiento productivo de cabras en el trópico seco de México. Memorias de la X Reunión Nacional sobre Caprinocultura; FMVZ-UAZ, AMPCA. Zacatecas Zac; 1995. p. 11-7.

2.- Wilkins JF, Crocker KP. Embryonic wastage in ewes En: Oldan CM, Martin MB, Porvis LW, editores. Reproductive Physiology of Merino Sheep, Concepts and Consequences. Perth: University of Western Australia; 1990. p. 169-7.

3.- Thatcher WW, Staples CR, Danet-Desnogers G, Oldick B, Schmitt EP. Embryo health and mortality in sheep and cattle. J Anim Sci 1994; 72 (Suppl 3):16-30.

4.- Baril G, Remy B, Leboeuf B, Beckers JF, Saumande, J. Synchronization of estrus in goats: the relationships between eCG binding in plasma, time of occurrence of estrus and fertility following artificial insemination. Theriogen 1996; 45:1553-9.

5.- Freitas VJF, Baril G, Saumande J. Induction and synchronization of estrus in goats: the relative efficiency of one versus two fluorogestone acetate-impregnated vaginal sponges. Theriogen 1996; 46:1251-6.

6.- Rueda MDF, Trejo GA, Salinas AR. Efecto de dosis de poligestona usadas como preparadores sobre la tasa ovulatoria y la prolificidad en cabras criollas desafiadas con PMSG para la ovulación múltiple durante el anestro. Memorias de la XII Reunión Nacional sobre Caprinocultura. UAAAN, Torreón Coahuila, Mex.; 1997. p. 103-8.

7.- Coteel JM. The use of progestagens to control oestrus cycle of the dairy goat. Ann Biol Anim Bioch Biophsys 1975; 15:353-63.

8.- Baril G, Leboeuf B, Saumande J. Synchronization of estrus in goats: the relationships between time of occurrence of estrus and fertility following artificial insemination. Theriogen 1993; 40:621-8.

9.- Kusina NT, Tarwirei F, Hamudikuwanda H, Agumba G, Mukwena JA. Comparation of the effects of progesterone sponges and ear implants, prostaglandins and their combination on efficacy of estrus synchronization and fertility of Mashona goat does. Theriogen 2000 53:1567-80.

\section{Revista Biomédica}

10.- Chávez GL, Zarco QL, Ducoing WA, Flores PG. Utilización de acetato de melengestrol y acetato de fluorogestona solos o combinados con gonadotropina sérica de yegua preñada para la sincronización de estros en cabras lecheras. AZTECA. Memorias del VII Congreso Nacional en Caprinocultura. Sinaloa, Mex.; 1990. p. 1478.

11.- Estrada BE, Gutiérrez AJ. Uso de Acetato de Fluorogestona, Norgestomet y Norgestometprostaglandinas en la sincronización de estros en cabras. Memorias de la XII Reunión Nacional sobre Caprinocultura, AMPCA, Torreón Coahuila; 1997. p. 191-5.

12.- Cervantes J, Ducoing WA, Flores PG, Zarco Q. Utilización del acetato de melengetrol y acetato de fluorogestona para la inducción de la pubertad en cabras primalas y para la inducción de estros durante la estación de anestro en cabras adultas. AZTECA. Memorias del V Congreso Nacional sobre Caprinocultura; UNAM, Mex.; 1988. p. 36-6.

13.- Torres-Acosta JF, Aguilar-Caballero A, OrtegaPacheco A. Comportamiento reproductivo de cabras criollas en el trópico subhúmedo del estado de Yucatán, México. XII Reunión Nacional sobre Caprinocultura. UAAAN, Torreón Coahuila, Mex.; 1997. p. 83-6.

14.- East NE, Rowe JD. Subcutaneous progestin implants vs. intavaginal sponges for dairy goats estrus synchronization during the transitional period. Theriogen 1989; 32: 921-8.

15.- Hulet CV. Determining fetal numbers in pregnant ewes. J Anim Sci 1977; 36:325-30.

16.- Bolet G. Timing and extent of embryonic mortality in pigs, sheep and goats; genetic variability. En: Sreenan JM, Diskin MG, editores. Embryonic Mortality in Farm Animals. Dordrecht: Martinus Nijhoff Publishers; 1986. p. 12-43.

17.- White DH, Rizzoli DJ, Cumming AI. Embryo survival in relation to number and site of ovulation in ewe. Aust J Exp Agric Anim Husb 1981; 21:32-8.

18.- Savio JD, Thatcher WW, Morris GR, Entwistle K, Drost M, Mattiacci MR. Effects of induction of low plasma progesterone concentration with a progesterone-releasing intravaginal device on follicular turnover and fertility in cattle. J Rep Fert 1993; 98:77-4. 